\title{
Comparison of selective media for the isolation of salmonellae
}

\author{
KEVIN ANDERSON AND HELEN KENNEDY \\ From the Salmonella Reference Laboratory, ${ }^{1}$ Institute of Medical and Veterinary \\ Science, Adelaide, South Australia
}

SYNOPSIS A study of the selective action of four fluid media used for the isolation of salmonellae is described. When incubated in the presence of three competing organisms, test strains were recovered most frequently from Rappaport medium but mannitol selenite broth proved superior for the isolation of $S$. typhi.

Experiments carried out to assess the efficiency of liquid selective media for the isolation of salmonellae are difficult to design. Many variables are encountered in routine diagnostic work and these include the nature of the material to be cultured, the random distribution of pathogens in the sample, and the possible toxic effect of the medium upon these organisms. Media have been described which have a specific use and which may assist in the isolation of one particular serotype. Hobbs and Allison (1945) found that mannitol selenite broth favoured the isolation of $S$. typhi, while Harvey and Price (1964) describe the use of lactose selenite broth containing $0.8 \%$ sodium hydrogen selenite for the isolation of this organism from polluted water. The observation that the addition of cystine to selenite broth gives better recoveries of salmonellae from egg products has been recorded by North and Bartram (1953).

Rappaport, Konforti, and Navon (1956) used an artificially infected faecal suspension to test their medium, but the types of competing organism were not investigated. In this series of experiments we have used a system in which salmonellae serotypes were grown in liquid selective media in competition with three organisms which are commonly found in faeces and also frequently appear on solid indicator medium after subcultures have been taken from the selective broth.

\section{MATERIALS AND METHODS}

SALMONELLA SEROTYPES The following strains representing the main antigenic groups were selected for this study:-Group A $S$. paratyphi $A$; group B $S$. paratyphi $B$

'Supported by a full-time grant from the National Health and Medical Research Council of Australia.

Received for publication 3 May 1965. and $S$. typhimurium; group $\mathrm{C} S$. paratyphi $C$ and $S$. cholerae suis; group D $S$. typhi; group E $S$. london; group $\mathrm{E}_{2} S$. newington; group $\mathrm{E}_{4} S$. senftenberg; other groups $S$. adelaide.

COMPETING ORGANiSMS Esch. coli, Ps. pyocyanea, and Proteus mirabilis were used.

SELECTIVE MEDIA Selenite broth (lactose or mannitol) and tetrathionate broth were prepared according to the formulae of Mackie and McCartney (1953). Rappaport medium was made according to the description given by Rappaport et al. (1956). All media were dispensed in $9 \mathrm{ml}$. amounts in narrow-necked Universal containers. Peptone water consisting of $1 \%$ peptone in $0.5 \%$ sodium chloride solution was used as a control medium throughout these experiments.

EXPERIMENTAL METHODS Overnight broth cultures of salmonellae were diluted tenfold in equal parts of peptone water and saline. Viable counts were performed by the method of Miles and Misra (1938) and dilutions of $10^{-5}, 10^{-6}$, and $10^{-7}$ were selected to give approximately $1,000,100$, and 10 organisms per $\mathrm{ml}$. Three sets of the four test media with peptone water controls were inoculated with $0.5 \mathrm{ml}$. of the prepared dilutions of a salmonella culture together with $0.5 \mathrm{ml}$. (approximately $10^{3}$ cells) of an overnight broth culture of one of the competing organisms. Containers were incubated for 22 hours at $37^{\circ} \mathrm{C}$. and then subcultured with a standard loop onto S.S. medium (Difco). Plates were examined after overnight incubation at $37^{\circ} \mathrm{C}$. Following a number of preliminary experiments, it was possible to assess the distribution of organisms with a considerable degree of accuracy. Colonies of $E$. coli were recognized by lactose fermentation, while the strain of Proteus was detected by urease activity. Colonies were picked with a straight wire and stabbed into plates of urea agar which were incubated for two hours at $37^{\circ} \mathrm{C}$. Colonies of Ps.pyocyanea were recognized by colonial morphology, pigment formation, and the oxidase reaction. The salmonella 
serotype under investigation was identified by slide agglutination with specific antisera. Plates were allotted a numerical score from 0 to 4 , relating to the number of salmonella colonies expressed as a percentage of the total growth distributed on the surface of the medium.

\begin{tabular}{ll} 
Percentage of & Score \\
Salmonella Colonies & \\
\hline $100-75$ & 4 \\
$74-50$ & 3 \\
$49-25$ & 2 \\
$24-1$ & 1 \\
0 & 0
\end{tabular}

\section{RESULTS}

A preliminary statistical analysis indicated that the results were not significantly influenced by the size of the inoculum. The scores obtained with the three dilutions of each serotype used in these experiments were therefore added together and are summarized in Tables I, II, and III. Adding the percentage selectivity of each medium for all salmonellae except $S$. typhi, observed in competition with the three contaminating organisms, Rappaport medium

TABLE I

SELECTIVITY OF MEDIA IN COMPETITION WITH E. COLI

Salmonella

Medium

Rappaport

Lactose Selenite

Mannitol Selenite

Tetrathionate

Peptone Water

S. paratyphi $A$

S. paratyphi $B$

S. typhimurium

S. paratyphi $C$

S. cholerae suis

S. typhi

S. london

$S$. newington

$S$. senftenberg

$S$. adelaide

Total scores

Selectivity for S. typhi (\%)

Selectivity for other Salmonellae (\%)

5
12
12
12
12
0
12
12
8
12
97
0
$89 \cdot 2$

0
2
0
1
6
0
0
1
0
11
21
0

TABLE II

SELECTIVITY OF MEDIA IN COMPETITION WITH PROTEUS MIRABILIS

Salmonella

Medium

Rappaport Lactose Selenite Mannitol Selenite

Tetrathionate

4
6
6
$9 \cdot 5$
$11 \cdot 5$
4
$8 \cdot 5$
10
10
$7 \cdot 5$
77
33,3
$67 \cdot 6$

Peptone Water

S. paratyphi $A$

S. paratyphi $B$

S. typhimurium

S. paratyphi $C$

S. cholerae suis

S. typhi

$S$. london

$S$. newington

$S$. senftenberg

$S$. adelaide

Total scores

Selectivity for S. typhi $(\%)$

Selectivity for other Salmonellae (\%)

12
12
12
12
12
11
12
12
12
12
119
$91 \cdot 7$
100

1
2
8
$7 \cdot 5$
9
12
4
4
5
12
$64 \cdot 5$
100
$48 \cdot 6$

0
4
9
$8 \cdot 5$
9
11
4
9
12
$5 \cdot 5$
72
$91 \cdot 7$
$56 \cdot 5$

TABLE III

SELECTIVITY OF MEDIA IN COMPETITION WITH PS. PYOCYANEA

Salmonella

Medium

\begin{tabular}{l} 
\\
\hline S. paratyphi $A$ \\
S. paratyphi $B$ \\
S. typhimurium \\
S. paratyphi $C$ \\
S. cholerae suis \\
S. typhi \\
S. london \\
S. newington \\
S. senftenberg \\
S. adelaide \\
Total scores \\
Selectivity for S. typhi $(\%)$ \\
Selectivity for other Salmonellae $(\%)$
\end{tabular}

Rappaport

Lactose Selenite

Mannitol Selenite

Tetrathionate

Peptone Water

$\begin{array}{lcc}0 & 0 & 0 \\ 0 & 0 & 0 \\ 4 & 2 & 4 \\ 0 & 0 & 0 \\ 2 & 3 & 3 \\ 5 & 0 & 3 \\ 0 & 0 & 0 \\ 6 & 5 & 1 \\ 5 & 3 & 2 \\ 5 & 5 & 3 \\ 27 & 18 & 16 \\ 41 \cdot 7 & 0 & 25 \\ 20 \cdot 4 & 16 \cdot 7 & 12\end{array}$


is found to be superior to the others. Tetrathionate broth is better than mannitol selenite broth, which in turn is more selective than lactose selenite broth. For the isolation of $S$. typhi, mannitol selenite broth is the most effective. These differences are statistically significant.

\section{DISCUSSION}

In the experimental system described, Rappaport medium is superior for the isolation of all salmonellae tested, with the exception of $S$. typhi. These results agree with the observations of Iveson, Kovacs, and Laurie (1964) working with desiccated coconut, and with those of Collard and Unwin (1958) using faecal specimens. Rappaport medium showed no inhibitory action on the group $E$ salmonellae used in these experiments, which is contrary to the experience of Collard and Unwin but in agreement with the work of Iveson et al. (1964). The superiority of mannitol selenite broth for the isolation of $S$. typhi is clearly demonstrated and confirms the observations of Hobbs and Allison. Liquid selective cultures are always used in combination with a solid indicator medium, which itself has an inhibitory action on the competing organisms. These results therefore represent the combined effects of two systems, one of which (S.S. medium) remained constant throughout. It is of interest that an inoculum ranging from approximately 5 to 500 salmonellae did not significantly influence the performance of the individual selective media, even in the presence of relatively large numbers of competing organisms. The amount and nature of the material added to the fluid selective media may change their selective efficiency, although Rappaport et al. found that the rate of multiplication of salmonellae added in faeces or saline was of the same order. The presence of large numbers of $\boldsymbol{P s}$. pyocyanea appears to have an adverse effect on salmonellae isolation in all media tested. Other strains of Esch. coli and Proteus may have a similar effect; even so, Rappaport medium is better than the rest. Colicines active against the competing strain could not be demonstrated.

It could be argued that an increase in selectivity may be accompanied by a greater degree of inhibition of the salmonella which it is hoped to recover on subculture. Consequently, media which are less efficient under the conditions of this experiment could be more useful in routine use provided that some colonies of the pathogen were obtained on the S.S. plate. However, if Rappaport medium is effective with an inoculum of approximately 5 cells, in the face of heavy contamination, it would appear that the degree of inhibition cannot be significantly great in these laboratory experiments. For routine diagnostic work, therefore, Rappaport medium may be the selective medium of choice, and, in our experience, has proved excellent for the culture of faeces, desiccated coconut, meat, and meat products. When $S$. typhi is sought, either in suspected carriers, convalescent cases, or other material, mannitol selenite broth should be used.

Our thanks are due to Dr. J. R. Coulter, of the Medical Research Division, Institute of Medical and Veterinary Science, for assistance with the statistical analysis of these results.

\section{REFERENCES}

Collard, P., and Unwin, M. (1958). J. clin. Path., 11, 426 .

Harvey, R. W. S., and Price, T. H. (1964). Mth. Bull. Minist. Hlth Lab. Serv., 23, 233.

Hobbs, B. C., and Allison, V. D. (1945). Ibid., 4, 63.

Iveson, J. B., Kovacs, N., and Laurie, Wm. (1964). J. clin. Path., 17. 75.

Mackie, T. J., and McCartney, J. E. (1953). Handbook of Practical Bacteriology 9th ed., pp. 184-185. Livingstone, Edinburgh.

Miles, A. A., and Misra, S. S., (1938) J. Hyg. (Lond.), 38, 732.

North, W. R., and Bartram, M. T. (1953). Appl. Microbiol., 1, 130.

Rappaport, F., Konforti, N., and Navon, B. (1956). J. clin. Path., 9, 261 . 